\title{
Silencing TAK1 reduces MAPKs-MMP2/9 expression to reduce inflammation-driven neurohistological disruption post spinal cord injury
}

Shuai Jiang ${ }^{1}$, Yandan $\mathrm{Wu}^{2}$, Shunjie $\mathrm{Wu}^{1}$, Suhui $\mathrm{Ye}^{1}$, Renyi Kong ${ }^{3}$, Jie Chang ${ }^{3}$, Mingjie Xia ${ }^{4}$, Junping Bao ${ }^{1}$, Xin Peng ${ }^{1}$, Xin Hong ${ }^{1}$, Zhanyang Qian ${ }^{1}$ and Haijun Li ${ }^{5}$

\begin{abstract}
Microglia activation post traumatic spinal cord injury (SCl) provokes accumulation of inflammatory metabolites, leading to increasing neurological disruption. Our previous studies demonstrated that blocking MAPKs pathway mitigated microglia inflammatory activation and prevented cords from neuroinflammation-induced secondary injury. Transforming growth factor- $\beta$-activated kinase 1 (TAK1) is an upstream gate regulating activation of MAPKs signaling. To validate the therapeutic effect of TAK1 inhibition in neuroinflammation post SCI, in the current study, cultures of microglia BV2 line was undergone lipopolysaccharide (LPS) stimulation in the presence of TAK1 inhibitor 5Z-7-Oxozeaenol (ZO), LPS, or control. LPS triggered inflammatory level, cell migration, and matrix metalloproteinase (MMP) 2/9 production, which was reduced in ZO-treated cultures. TAK1 inhibition by ZO also decreased activation of MAPKs pathway, indicating that ZO-mediated alleviation of neuroinflammation is likely modulated via TAK1/MAPKs axis. In vivo, neuroinflammatory level and tissue destruction were assessed in adult male mice that were undergone SCI by mechanical trauma, and treated with ZO by intraperitoneal injection. Compared with SCl mice, ZO-treated mice exhibited less microglia pro-inflammatory activation and accumulation adjacent to injured core linked to reduced MMP2/9 expression, leading to minor tissue damage and better locomotor recovery. To sum up, the obtained data proved that in the early phase post SCI, TAK1 inhibition impedes microglia biological activities including activation, enzymatic synthesis, and migration via downregulation of MAPKs pathway, and the effects may be accurately characterized as potent anti-inflammation.
\end{abstract}

\section{Introduction}

Microglia, as a resident immune cell in neural tissue, plays a vital role in inflammatory and immune responses post neurological injury ${ }^{1,2}$. Following a traumatic spinal cord injury (SCI) inducing primary mechanical contusion of neural tissue, resting microglia rapidly activate and rally to the lesion with overproduction of pro-inflammatory factors to stimulate neuroinflammation ${ }^{3-5}$. Next,

Correspondence: Xin Hong (xinhong102400@163.com) or

Zhanyang Qian (spineqzy@126.com) or Haijun Li (13901436563@139.com) ${ }^{1}$ Spine Center, Zhongda Hospital of Southeast University, Nanjing, China

2Department of Microbiology and Immunology, Medical School, Southeast University, Nanjing, China

Full list of author information is available at the end of the article These authors contributed equally: Shuai Jiang, Yandan Wu, Shunjie Wu Edited by Richard Killick activated microglia works together with infiltrating leukocytes from the peripheral circulation to release an increased quantity of pro-inflammatory mediators and reactive oxygen species (ROS) while allowing higher infiltration of peripheral leukocytes to exacerbating secondary tissue injury surrounding the primary lesion ${ }^{6}$. The current clinical treatment for SCI is still administrated large dose of methylprednisolone, but increasing researchers have suggested that the routine ictus treatment not only did not significantly inhibit the acute neuroinflammation, but also the side effects of treatment had a great burden on the patient's body ${ }^{7,8}$. How to effectively increase control over acute neuroinflammation post traumatic nerve injury to improve tissue repair is a contentious unresolved issue.

\section{(c) The Author(s) 2021}

(c) (i) Open Access This article is licensed under a Creative Commons Attribution 4.0 International License, which permits use, sharing, adaptation, distribution and reproduction cc) in any medium or format, as long as you give appropriate credit to the original author(s) and the source, provide a link to the Creative Commons license, and indicate if changes were made. The images or other third party material in this article are included in the article's Creative Commons license, unless indicated otherwise in a credit line to the material. If material is not included in the article's Creative Commons license and your intended use is not permitted by statutory regulation or exceeds the permitted use, you will need to obtain permission directly from the copyright holder. To view a copy of this license, visit http://creativecommons.org/licenses/by/4.0/. 
Transforming growth factor- $\beta$-activated kinase (TAK1) is originally defined as a mitogen-activated protein kinase kinase kinase (MAP3K) activated by TGF- $\beta^{9}$. Since this crucial report, TAK1 subsequently has been found to regulate various signaling pathways, including inflammation, apoptosis, and oxidative stress ${ }^{10-12}$. In inflammatory response, TAK1 activation by microbial lipopolysaccharide (LPS), TNF- $\alpha$, and IL-1 phosphorylates the complex of $\mathrm{IKK}$, which further degrades $\mathrm{I} \kappa \mathrm{B}$, leading to activation of NF- $\mathrm{KB}^{13-15}$. Phosphorylation of TAK1 also activates MAPKKs, resulting in activation of MAPKs signaling pathways $^{16}$. ZO is a classic TAK1 irreversible inhibitor through forming a covalent complex with TAK $1^{17}$. It was reported that $\mathrm{ZO}$ attenuates the development of inflammation and tissue damage in a number of experimental disease models ${ }^{17-19}$. Given the previous presence, we hypothesize that inhibition of TAK1 by $\mathrm{ZO}$ serves as an effective targeted therapy against microglia-induced neuroinflammation and oxidative stress post SCI.

In the current study, we verified a previously unidentified effect for TAK1 inhibition on microglia-induced neuroinflammation and tissue degradation after SCI. Also, a potent target was found for control over neuroinflammation post SCI through the MAPKs signaling pathways. Meaningfully, our findings systematically display that suppression of microglia-driven neuroinflammation exerts important functions on protection against secondary injury and promotion for further pathological healing during the progression of SCI.

\section{Results}

High phosphorylation of TAK1 and p38 in vivo and in vitro during neuroinflammation

To elucidate whether TAK1 activation involves in neuroinflammatory response, the expression of phosphorylated TAK1 (p-TAK1) was measured in vivo SCI models and in vitro LPS-treated microglia by WB after $24 \mathrm{~h}$. It was found that phosphorylated TAK1 increased significantly following SCI modeling in mice and LPS treatment in microglia (Fig. 1A-D). Furthermore, the activation of a downstream signaling conductor p38 was visualized using IF, showing that p38 was markedly activated in injured cord, especially in ionized calcium-binding adaptor protein 1 (IBA1) labeled microglia (Fig. 1E). In vitro, high expression of phosphorylated p38 (p-p38) was also observed in LPS-mediated microglial neuroinflammation (Fig. 1F). The results showed that extensive activations of TAK1 and p38 were witnessed in neuroinflammatory microglia both in vivo and in vitro.

\section{Inhibition of TAK1 blocks pro-inflammatory transition of microglia by reducing MAPKs pathway}

After LPS treatment for $24 \mathrm{~h}$, inducible nitric oxide synthase (iNOS), a pro-inflammatory marker of microglia, and p-p38 were examined by IF. Interestingly, we found that neuroinflammatory activation increased iNOS and pp38 expressions in microglia yet inhibition of TAK1 by ZO treatment reduced iNOS and p-p38 protein levels in activated cells (Fig. 2A). Moreover, WB showed that activation of other inflammation-related pathways, including $\mathrm{I}_{\kappa} \mathrm{B} \alpha$, JNK1/2, and ERK1/2, were decreased after TAK1 inhibition during neuroinflammatory response as well (Fig. 2B, C). Importantly, reduced expression of p65 was presented in LPS-treated microglia after $\mathrm{ZO}$ employment (Fig. 2D). FCA results exhibited a dramatic increase in F4_80-PE positive and iNOS-FITC positive cells following LPS stimulation but a remarkable inhibition of iNOS-FITC positive microglia ratio by $\mathrm{ZO}$ administration (Fig. 2E, F), indicating that decreased TAK1 activation inhibited inflammatory phenotype of microglia. It was shown that the RNA expressions of classic inflammatory cytokines, such as tumor necrosis factor (TNF)- $\alpha$ and interleukin (IL) $-1 \beta$, prominently decreased in TAK1inhibited microglia after LPS utilization for $12 \mathrm{~h}$ (Fig. 2G). Consistently, IF displayed that the protein expressions of TNF- $\alpha$ and IL-1 $\beta$ were reduced in activated microglia under TAK1 suppression after LPS treatment for $24 \mathrm{~h}$ (Fig. 2H). Collectively, blocking TAK1 attenuates microglial inflammation via inhibiting MAPKs-related signaling axis.

\section{Disruption of TAK1 reduces microglia migration and matrix metalloproteinases (MMPs) production}

To verify whether restraining TAK1 affects inflammation-driven microglial migration, trans-well assay was carried out to evaluate the capacity of microglia. As shown in Fig. 3A, LPS-mediated inflammation provoked more migrated microglia, while TAK1 inhibition by $\mathrm{ZO}$ significantly decreased the amount of microglia compared with the LPS group (Fig. 3B). Besides, the representative chemokine monocyte chemoattractant protein-1 (MCP-1) was found to be increased signally after LPS treatment. However, disrupting TAK1 activation reduced MCP-1 expression (Fig. 3C). MMPs generation was measured at the levels of both transcription and translation. Results showed that the RNA and protein levels of MMP2 and MMP9 elevated by LPS inducement, whereas $\mathrm{ZO}$ employment inhibited their expressions during microglial inflammation (Fig. 3D, E). Taken together, TAK1 blocking suppresses microglia migration and MMPs production during inflammatory response.

\section{TAK1 inhibition alleviates microglia-induced} neuroinflammation and MMP-induced tissue destruction

At 3 days post SCI (dpi), the inflammatory phenotype and the amount of microglia (longitudinal sections centered around the injured core $1 \mathrm{~mm}$ ) was measured using 
A

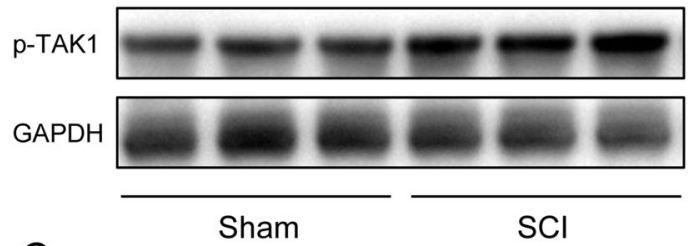

C
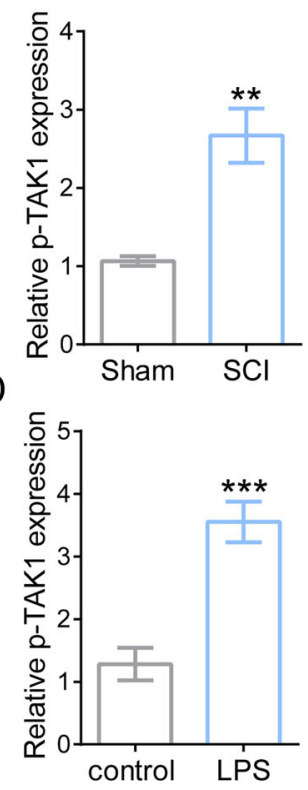

E
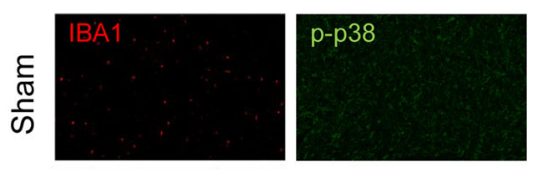

$\bar{c}$

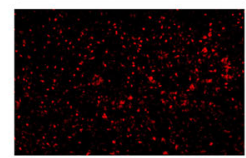

$\mathrm{F}$

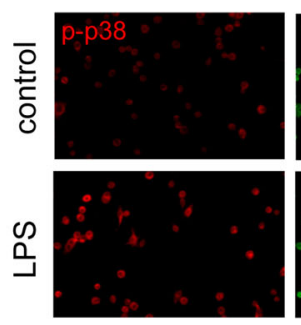

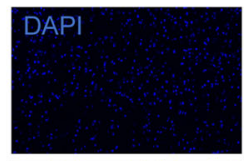
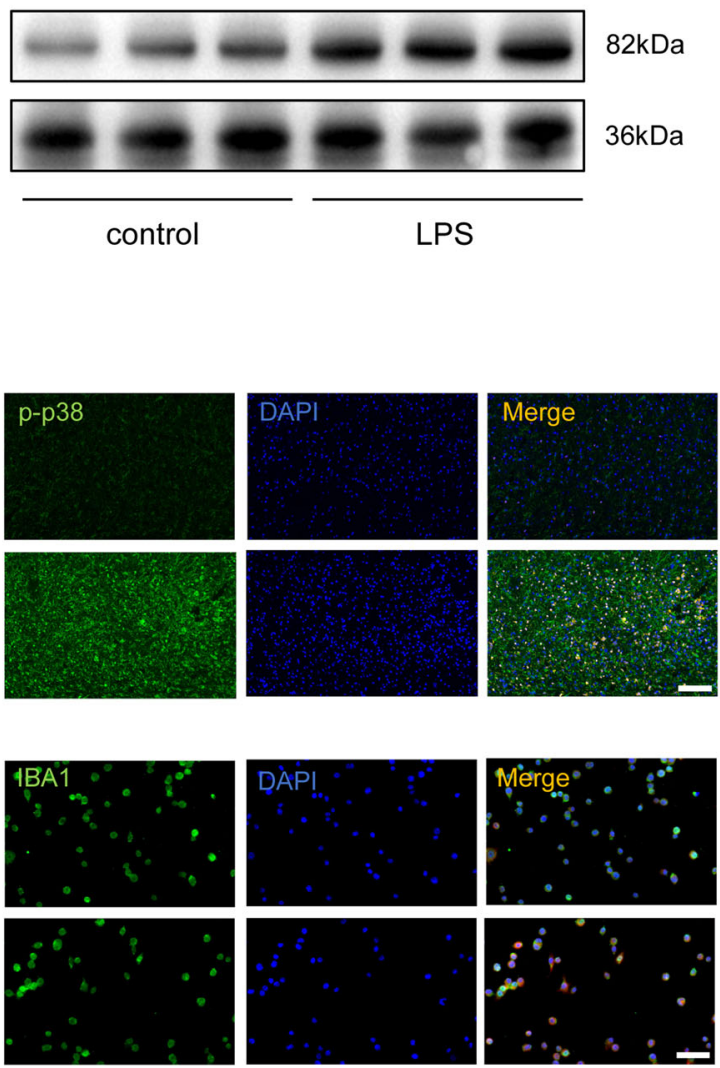

Fig. 1 The expression of phosphorylation of TAK1 and p38 in vivo and in vitro increased during neuroinflammation response. Western blotting of phosphorylated TAK1 ( $p$-TAK1) in vivo SCI model $\mathbf{A}$ and in vitro LPS-treated microglia (B). C Quantification of $p-T A K 1$ in vivo. ${ }^{* *} P<0.01$ vs. sham group; $n=6$ in each group. Data were collected in three independent experiments. D Quantification of $p-T A K 1$ in vitro. ${ }^{* * *} P<0.001$ versus control. Data are presented as mean $\pm \mathrm{SD}, n=6$ for each bar. E Representative IF images of phosphorylated p38(p-p38) in the injured cord; Bar scale $=100 \mu \mathrm{m}$. F Representative IF images of p-p38 were in LPS-mediated microglial neuroinflammation; Bar scale $=50 \mu \mathrm{m}$.

IF, showing that IBA1-positive area increased after SCI while TAK1 inhibition markedly reduced the accumulation of microglia (Fig. 4A, B). More notably, the cells costaining by IBA1 and iNOS exhibited a decrease in SCI mice under TAK1 inhibition, implying that TAK1 suppression reduced inflammatory microglia activation post SCI (Fig. 4A). Moreover, the inflammatory cytokines and chemokine, including TNF- $\alpha$, IL- $1 \beta$, and MCP-1, were examined using ELISA, exhibiting that blocking TAK1by $\mathrm{ZO}$ administration decreased their protein levels in SCI mice (Fig. 4C-E). WB analysis showed an increase in MAPKs pathways, including p-JNK1/2, p-ERK1/2, and p-p38 post SCI, whereas decreased trends of them were observed in injured cords after TAK1 invalidation (Fig. 4F, G). The expressions of MMP2 and MMP9 in injured cords were detected using IF, showing that SCI causes the excess generation of MMP2/9 yet TAK1 inhibition post SCI alleviated MMP2 and MMP9 expressions in SCI mice (Fig. 4H). Histologically, HE staining exhibited more severe destruction of cord tissue in SCI mice compared with those in $\mathrm{SCI}+\mathrm{ZO}$ group as well (Fig. 4I, J). These all results demonstrated that TAK1 inactivation prevents injured cords from tissue destruction caused by microglia-induced neuroinflammation and MMPs generation.

\section{TAK1 inhibition reduces glial forming and improves neurological recovery in $\mathrm{SCl}$ mice}

At $28 \mathrm{dpi}$, the glial scar components (IBA1 and GFAP labeled) and axons (NF200 labeled) were presented using tricolor IF staining. As shown in Fig. 5Ab, minor areas of microglia (pink) and astrocytes (red) and some axons (green) were witnessed in spinal cord tissue (longitudinal sections centered around the injured core $2 \mathrm{~mm}$ in Fig. 5Aa). In SCI group, a mass of glial scar composed of microglia and astrocytes occupied most of the spinal cord area with almost nonexistent axons. However, it was found with less glial scar area and some regenerated axons in SCI mice treated with TAK1 inhibition (Fig. 5A-C). From a perspective on histology, a less glial infiltration 
A
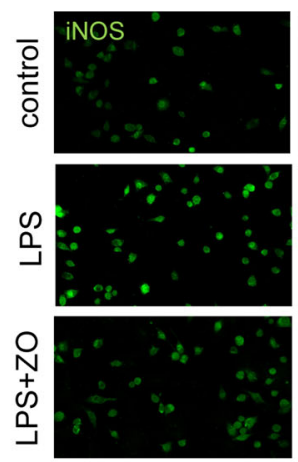

C

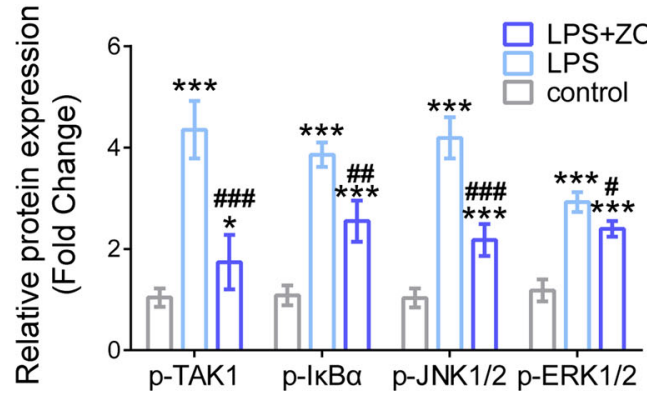

$\mathrm{E}$
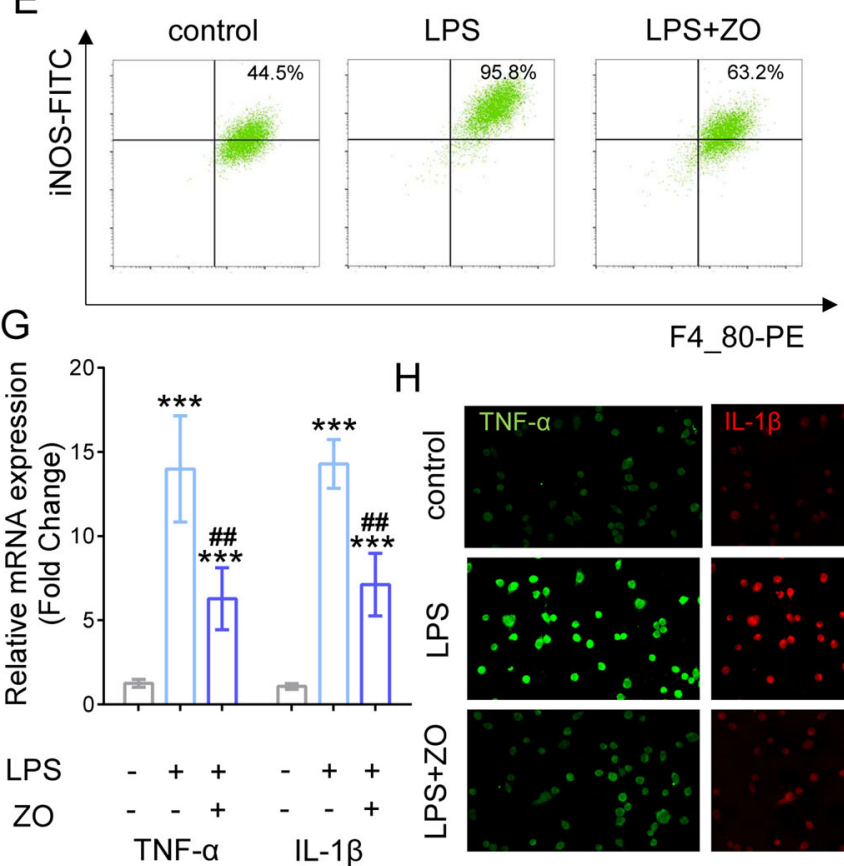
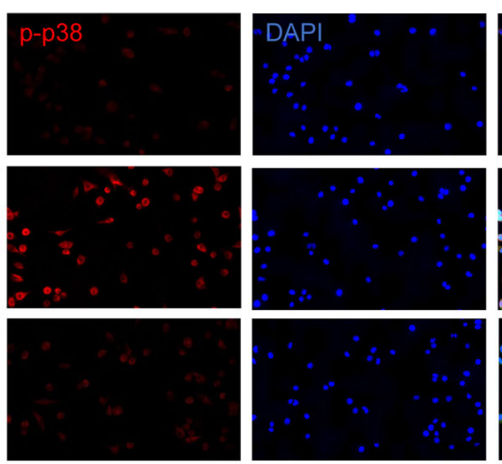

D
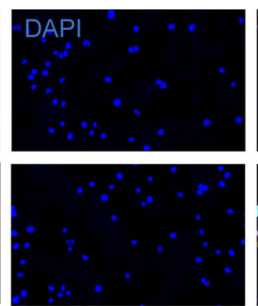

H

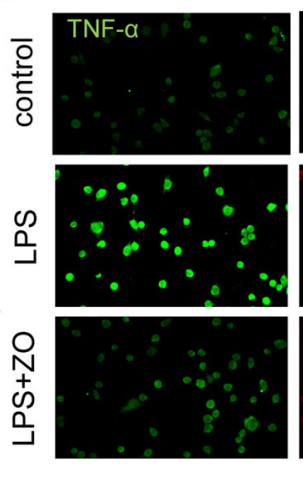

B
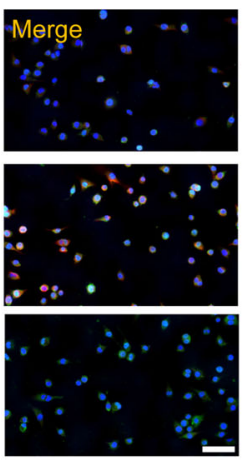

control
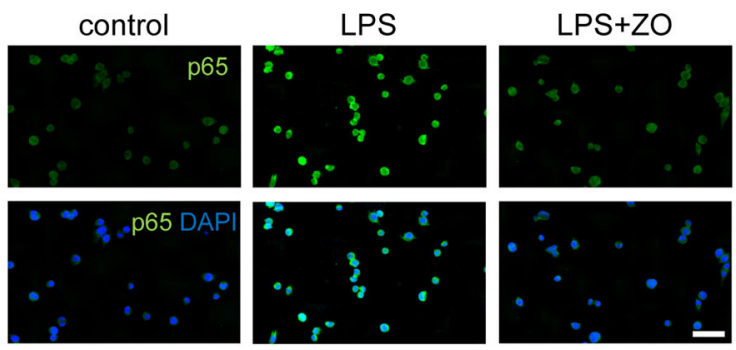

F

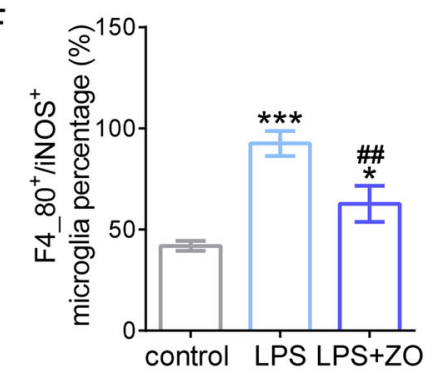

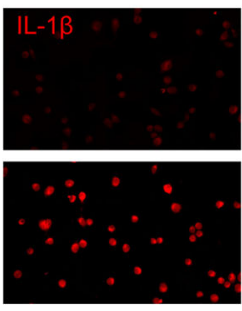
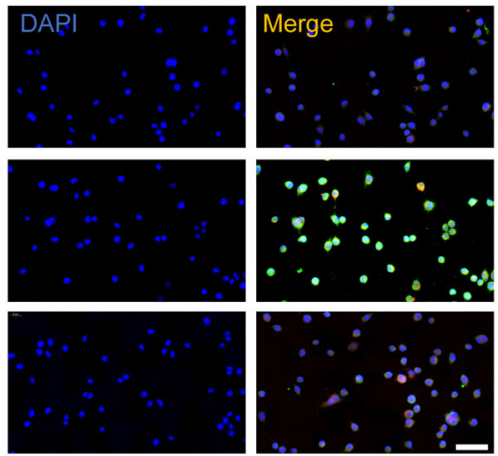

Fig. 2 Blocking TAK1 attenuates microglial inflammation by reducing MAPKs pathway. A Representative IF images of inducible nitric oxide synthase (iNOS) and p-p38; Bar scale $=50 \mu \mathrm{m}$. B Western blotting of phosphorylation of TAK1, IkBa (p-IkBa), JNK1/2 (p-JNK1/2), and ERK1/2 (pERK1/2) after TAK1 inhibition. C Quantification of p-TAK1, p-IKBa, $p$-JNK1/2, and $p$ - ERK1/2. ${ }^{*} P<0.05,{ }^{* * *} P<0.001$ versus control, \#P<0.05, \#\# $P<0.01$, \#\#\# $P<0.001$ versus LPS group. D Representative IF images of p65 in LPS-treated microglia after TAK1 inhibition; Bar scale $=50 \mu \mathrm{m}$. E FCA results of F4_80-PE positive and iNOS-FITC positive cells. F Quantification of iNOS-FITC positive microglia ratio after ZO administration. G Quantification of tumor necrosis factor (TNF)- $a$ and interleukin (IL)-1 $\beta$. H Representative IF images of TNF- $a$ and IL-1 $\beta$ under TAK1 inhibition after LPS treatment; Bar scale $=50 \mu \mathrm{m}$. 
B

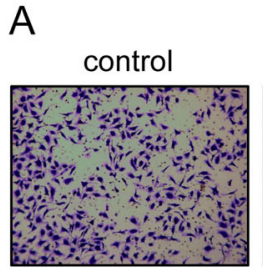

A

D

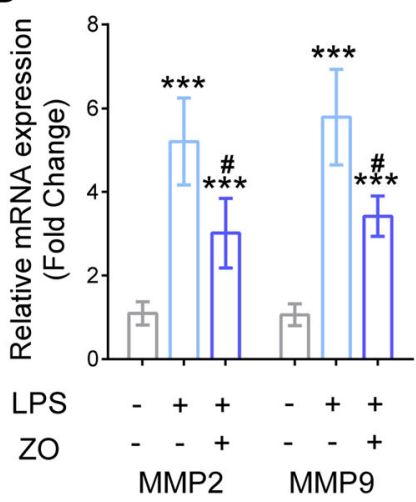

E
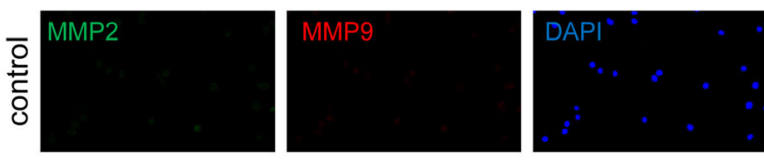

Merge
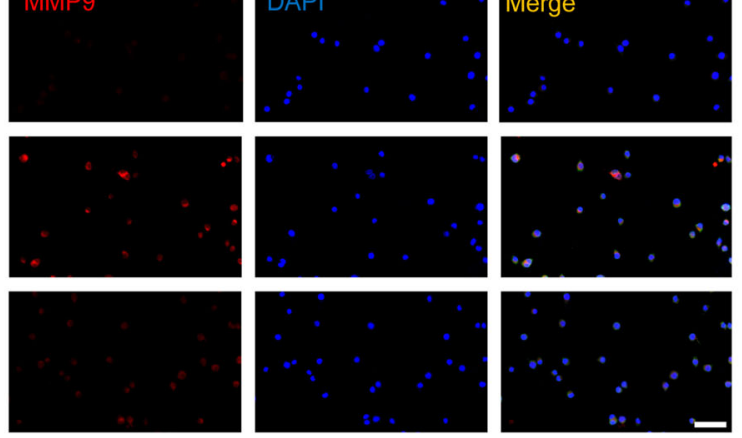

Fig. 3 Disruption of TAK1 inhibits microglia migration and MMPs production. A Trans-well assay with LPS and ZO administration treated as indicated. B The amount of microglia under LPS and TAK1 inhibition treatment. *** $P<0.001$ versus control, \# $P<0.05$ versus LPS group. C Quantification of Monocyte chemoattractant protein-1 (MCP-1). *** $P<0.001$ versus control, \# $P<0.05$ versus LPS group. D The RNA levels of MMP2 and MMP9 after LPS inducement and ZO employment. ${ }^{* *} P<0.001$ versus control, \# $P<0.05$ versus LPS group. E Representative IF images of MMP2 and MMP9; Bar scale $=50 \mu \mathrm{m}$.

around the injured cord and better tissue integrity was observed in TAK1 inhibitor-treated spinal cord (Fig. 5D, E). Functionally, based on the evaluation in accordance with BMS score, the locomotor recovery of the hindlimb in SCI mice was significantly improved by TAK1 inhibition, which appeared at $7 \mathrm{dpi}$ and then peaked at $28 \mathrm{dpi}$ (Fig. 5E). To sum up, blocking TAK1 activation ameliorates histological structure and benefits to neurological recovery post SCI.

\section{Discussion}

Mechanical contusion in the spinal cord causes a string of pathophysiological cascades launching secondary SCI. Thereinto, neuroinflammation voluntarily activates in response to primary injury ${ }^{20,21}$. However, excess inflammatory impact from microglia in situ triggers extensive destruction of histology and deterioration of motor function in the context of $\mathrm{SCI}^{22-24}$. Given its crucial clinical implications, it is desired for seeking a promising target to control over post-SCI neuroinflammation. Herein, our research concentrated on the attenuation of microgliaderived neuroinflammation and the preservation of neurological integrity by pharmacological inhibition of TAK1.

TAK1, encoded from the MAP3K7 gene, is located at the cellular hub where TNF- $\alpha$, TGF- $\beta$, and Wnt axis converge $^{25,26}$. To date, TAK1 has been certified as an essential interchanger of inflammatory response in various disease models. The reduction of TAK1 prevents lung inflammation in pneumoconiosis and reduces monosodium urate-induced inflammation as well as obesity-linked inflammation ${ }^{18,27,28}$; however, its biological function was little reported in neurological lesion especially in SCI. It is universally known that TAK 1 is activated by a component composed of TAK1-binding protein 2 (TAB2) and RIPK1 polyubiquitin chain. Then activated TAK1 further phosphorylates IKK $\alpha / \mathrm{IKK} \beta / \mathrm{IKK} \gamma$ complex, which degrades IKB protein, leading to NF- $\mathrm{kB}$ activation ${ }^{29,30}$. On the other hand, TAK1 activation promotes phosphorylation of MAPKKs, resulting in activation of MAPKs pathway, including JNK, ERK, and $\mathrm{p} 38^{31}$. NF- $\mathrm{KB}$ and MAPKs jointly incur downstream generation of inflammatory substances and degrading enzymes such as $\mathrm{MMPs}^{32,33}$. Our findings showed that p-TAK1 level was increased, along with p38 activation, in injured spinal cords and cultured BV2 microglia after modeling for $24 \mathrm{~h}$. Interestingly, $\mathrm{ZO}$ selectively inhibited phosphorylation of TAK1 and p38 in microglia, and reduced iNOS expression as well as the amount of pro-inflammatory cells. These results suggested a cellular mechanism that TAK1 inhibition partly blocked MAPKs pathway to decrease microglial response to inflammatory mediators. 
A
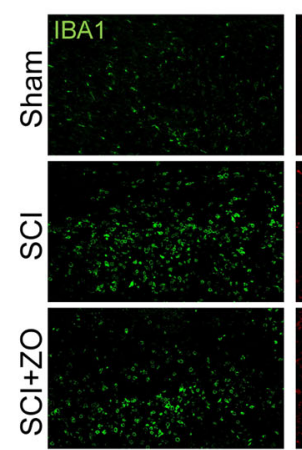

C

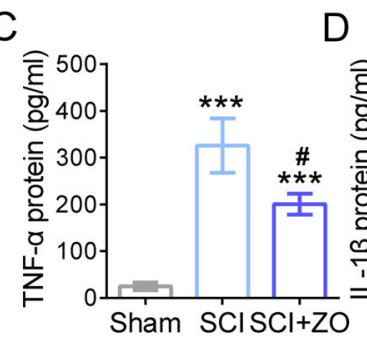

$\mathrm{G}$

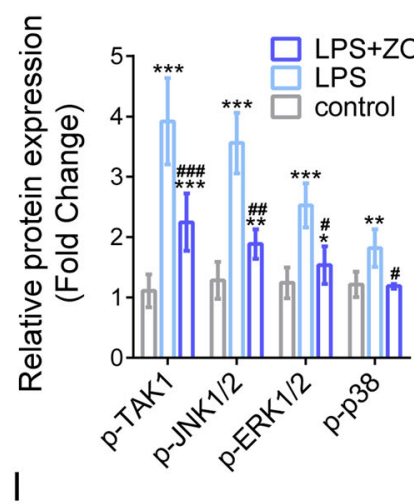

Sham

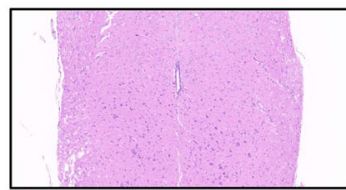

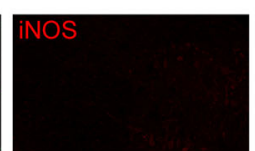
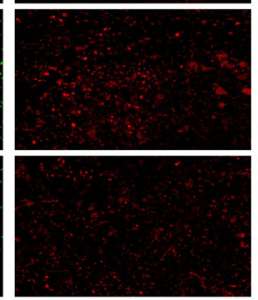

D

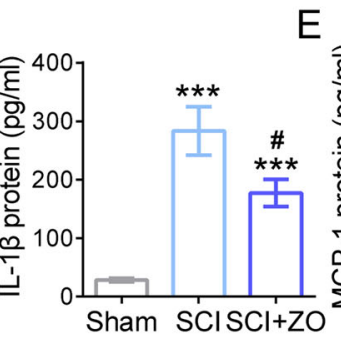

$\mathrm{H}$
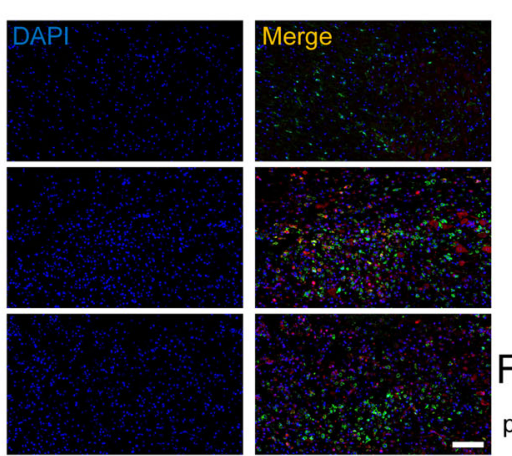

E
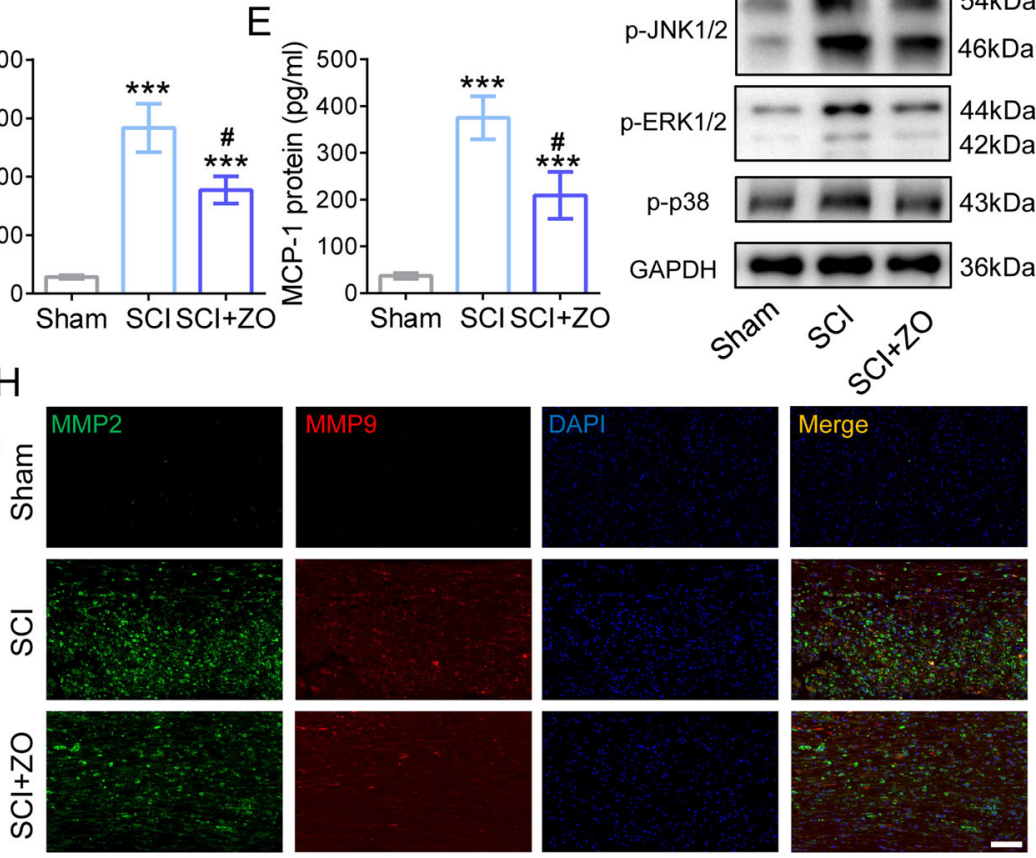

J

$\mathrm{SCl}$
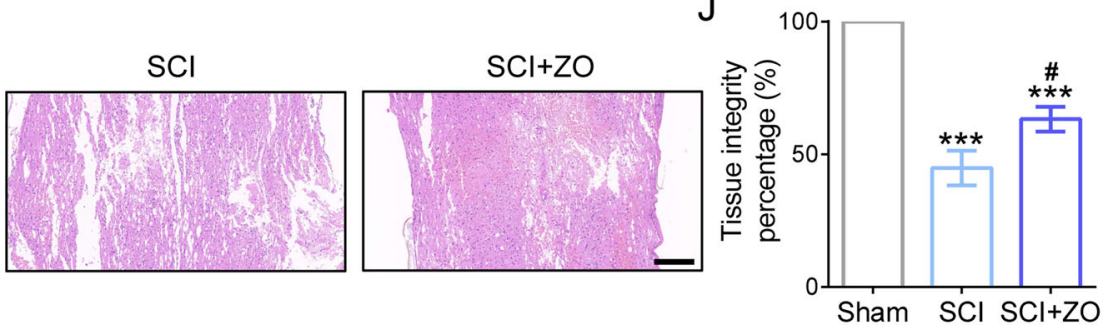

Fig. 4 TAK1 inhibition ameliorates neuroinflammation and tissue destruction caused by microglia and MMPs generation. A Representative IF images of IBA1 and iNOS after LPS inducement and ZO administration; Bar scale $=100 \mu \mathrm{m}$. B The amount of microglia under LPS and TAK1 inhibition treatment. ${ }^{* * *} P<0.001$ versus Sham group, $\# P<0.05$ versus $\mathrm{SCl}$ group. The protein levels of TNF- $\mathrm{a}$, IL-1 $\beta$, and $\mathrm{MCP}-1 .{ }^{* * *} P<0.001$ vs. Sham group, \# $P<0.05$ vs. SCl group C-E. F Western blotting of $\mathrm{p}-J \mathrm{NK} 1 / 2, \mathrm{p}$-ERK1/2 and $\mathrm{p}-\mathrm{p} 38$. G Quantification of $\mathrm{p}-\mathrm{JNK} 1 / 2, \mathrm{p}-\mathrm{ERK} 1 / 2$, and $\mathrm{p}-\mathrm{p} 38$. ${ }^{*} P<0.05$, ${ }^{* * *} P<0.001$ versus Sham group, \#P<0.05, \#\# $P<0.01$, \#\#\# $P<0.001$ versus SCl group. $\mathbf{H}$ Representative IF images of the expressions of MMP2 and MMP9 in injured cords; Bar scale $=100 \mu \mathrm{m}$. I Representative HE staining photos of spinal cord in Sham, SCl, SCl $+\mathrm{ZO}$ group at $3 \mathrm{dpi}$; Bar scale $=$ $200 \mu \mathrm{m}$. J Quantification of tissue integrity in the spinal cord.

MAPKs pathway represents an indispensable link in inflammatory signaling so that targeted regulation for MAPKs pathway mitigates advance of multiple diseases under a circumstance of inflammation, including cancer, autoimmune disease, and infectious disease ${ }^{34-36}$. Our previous studies logically reported pharmacological or 

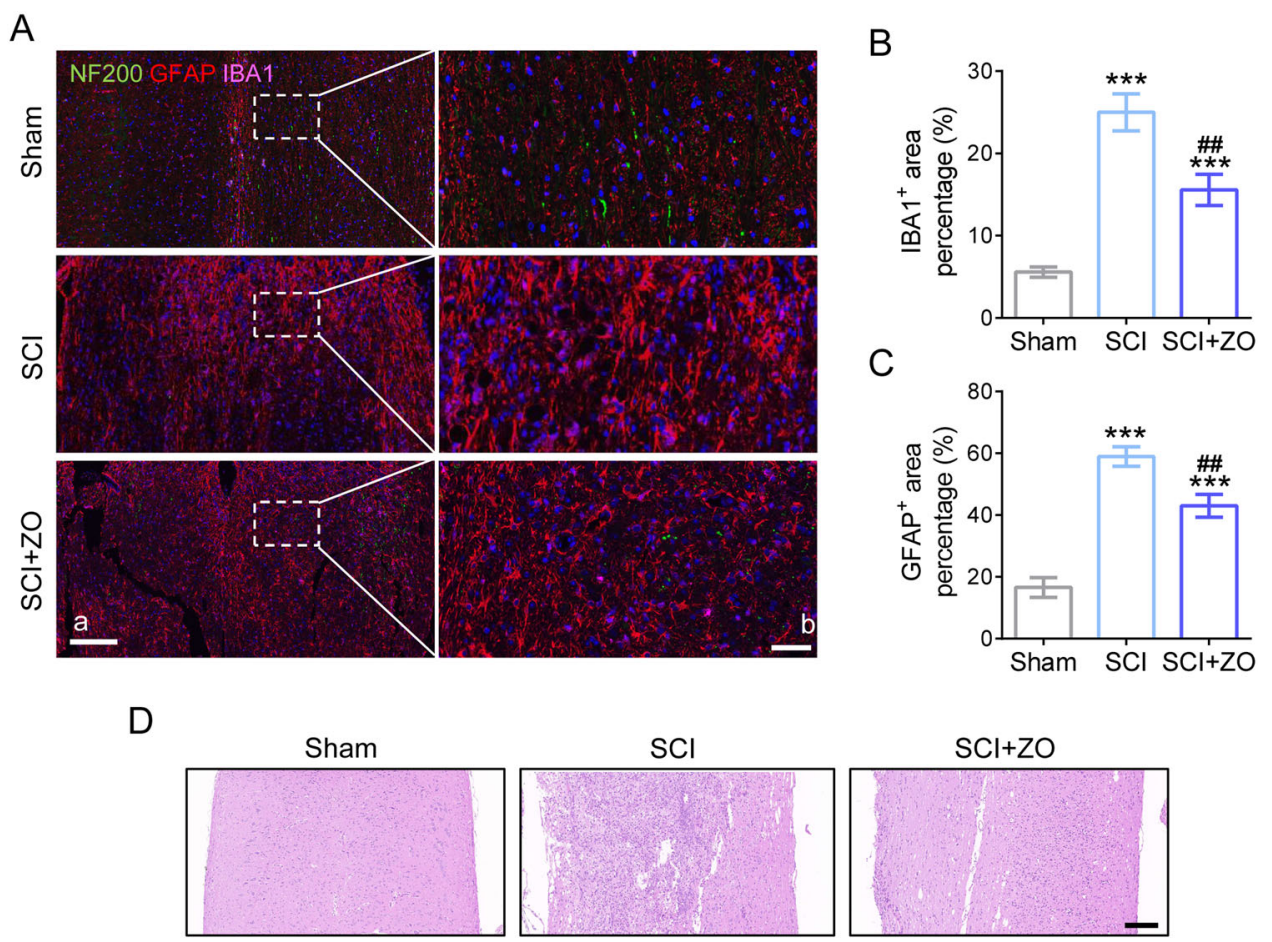

E

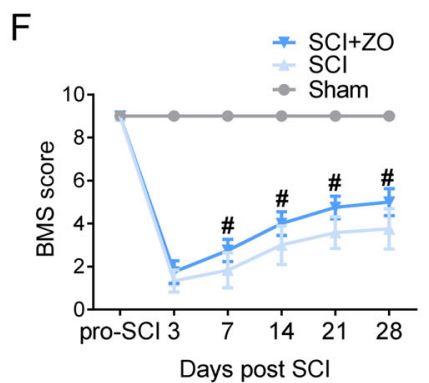

Fig. 5 TAK1 inhibition exerts a beneficial effect on histological structure and neurological recovery in SCI mice. A Trinary immunofluorescence shows the distribution of NF200 (green), GFAP (red), and IBA1 (pink) in the lesion site of the spinal cord at $28 \mathrm{dpi}$; a, Scale bar = $200 \mu \mathrm{m} ; \mathrm{b}$, Scale bar $=50 \mu \mathrm{m}$. B Quantification of the IBA1-positive area in the spinal cord at 28 days post-SCl. ${ }^{* * *} P<0.001$ versus Sham group, \#\# $P<$ 0.01 versus SCl group; $n=6$ in each group. C Quantification of the GFAP-positive area in the spinal cord at 28 days post-SCl. D Representative HE staining photos of the spinal cord in Sham, SCl, SCl + ZO group at 28 dpi; Bar scale $=200 \mu \mathrm{m}$. E Quantification of tissue integrity in the spinal cord. F The BMS score post $\mathrm{SCl}, \# P<0.05$ versus $\mathrm{SCl}$ group.

genetic interventions towards MAPKs pathway corrected skeletal muscle fibrosis and SCI as well ${ }^{37,38}$. We here found that TAK1, as an upstream target of MAPKs pathway, plays a potent regulation for microglia-induced neuroinflammation post SCI. As shown in our data, activation of not only p38 but also JNK and ERK was significantly inhibited after TAK1 inactivation in vitro and in vivo. Thus, reduced inflammatory cytokines like TNF- $\alpha$ and IL-1 $\beta$ were witnessed in microglia during neuroinflammation. It is non-negligible that microglia migrate from the distal to the proximal of injured core in that the process concurrently generates redundant inflammatory cytokines and chemokines for summon of glial cells and peripheric cells, such as astrocytes, macrophages, and neutrophils, instead of functional neural cells. In parallel studies worked in vitro, microglia migration aggravated when TAK1 was activated but attenuated as TAK1 inhibited by ZO. More notably, the employment of ZO to pharmacologically interfere with TAK1 activation concurrently mitigated MCP-1 expression.

MMPs are a type of enzymes that dissolve extracellular matrix under inflammation, tumor, and other pathological conditions $^{39,40}$. In the early phase of inflammation, outbreak of inflammatory cytokines promotes MMPs expression, which afterwards destructs basement-membrane for infiltration of inflammatory cells into deeper or larger tissue. Undoubtedly, microglia produce massive MMPs to exacerbate neural tissue destruction when activation in 
inflammatory state ${ }^{41}$. MMP2/9 have been widely identified as a group of agitators in tumor metastasis by promoting matrix breakdown to accelerate cell migration when proinflammatory mediators stimulated ${ }^{42,43}$. Activation of NF$\kappa \mathrm{B}$ and MAPKs pathways including JNK, ERK, and p38 upregulates MMP2/9 expression; however, selectively inhibiting one of them leads to a reduction of MMP2/9 production $^{44,45}$. Evidence showed that the microglial inflammatory state facilitates roles of matrix-dissolving enzymes for migration ${ }^{46}$. Naturally, through inhibiting neuroinflammation by TAK1 blocking in microglia, we observed a disruptive MMP2/9 production after SCI. The present study associated TAK1/MAPKs axis with microglial biological activity in neuroinflammation, in which TAK1 inhibition alleviated microglia migration along both lines, reducing MCP-1 level and blocking MMP2/9 expression. Also, selectively inhibition of TAK1 prevented severe histological loss at acute inflammatory period post $\mathrm{SCI}$ and possibly achieved better recovery of cord histology and function in ZO-treated mice than the untreated mice.

Taken together, TAK1inhibition exhibited a valid suppression of MAPKs activation and control of inflammatory arousal in microglia. However, indiscriminative pharmacological inhibition of TAK1 cannot thoroughly testify the specific superiority and inferiority in other neural cells. In addition, TAK1 is reported to involve in the development of apoptosis, oxidative stress, and autophagy $^{10,47}$, comprehensive and in-depth studies thereby need to be carried out for systematic evaluation of TAK1 in SCI treatment.

\section{Materials and methods}

\section{Reagent and antibodies}

ZO and LPS were obtained from MedChemExpress (Shanghai, China) and the culture medium was purchased from Servicebio (Wuhan, China). Fetal bovine serum (FBS) and Mouse ELISA Kits were purchased from Invitrogen (Carlsbad, CA, USA). Hematoxylin-eosin (HE) staining kit (G1005) and 4\% paraformaldehyde (PFA) were purchased from Servicebio. Dimethylsulfoxide (DMSO) was purchased from Solarbio (Beijing, China). TRIzol was purchased from YiFeiXue Biotech (Nanjing, China).

Anti-p-TAK1 (\#9339; 1:1000 for western blot (WB)), Anti-p-p38 (\#4511; 1:1000 for WB; immunofluorescence

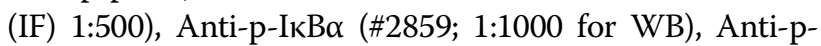
ERK1/2 (\#4370; 1:1000 for WB), Anti-JNK1/2 (\#4695; 1:1000 for WB), Anti-p65 (\#8242; 1:600 for IF), Anti-GFAP (\#3670; 1:600 for IF) were from Cell Signaling Technology (Danvers, MA, USA). Anti-IBA1 (\#ab178847; 1:500 for IF), Anti-iNOS (\#ab15323; 1:100 for IF), Anti-neurofilament heavy polypeptide (NF-200; \#ab207176; 1:200 for IF), AntiMMP2 (ab92536; 1:250 for IF), Anti-MMP9 (ab38898; 1:300 for IF) were from Abcam (Cambridge, MA, USA). Anti-F4_80-PE (565410) and iNOS-FITC (610330) were from BD Biosciences (Franklin Lakes, NJ, USA). Alexa Fluor 488-, 555- and 647-conjuated secondary antibodies (1:500 for IF) was from Jackson (Philadelphia, PA, USA). Anti-GAPDH (HRP-60004; 1:10000 for WB), HRPconjugated secondary antibodies (1:10000 for WB) were from Proteintech (Chicago, IL, USA).

\section{Cell culture and treatment}

A BV2 microglia line was obtained from the Institute of Cell Research, Chinese Academy of Medical Sciences Medical Science and cultured with Dulbecco's modified Eagle medium (DMEM) containing 10\% FBS in a cell incubator at $37^{\circ} \mathrm{C}$ with $5 \% \mathrm{CO}_{2}$. Microglia were carried out starvation after confluence reached $80 \%$, followed by pretreatment with $\mathrm{ZO}(1 \mu \mathrm{M}$, dissolved in $0.1 \%$ DMSO $)$ for $2 \mathrm{~h}$. Then LPS $(100 \mathrm{ng} / \mathrm{mL})$ was employed to stimulate microglia activation for a required time.

\section{$\mathrm{SCl}$ in mice}

Male adult C57BL/6J mice (average weight $20 \mathrm{~g}$ ) were obtained from the Experimental Animal Center of Nanjing Medical University and maintained in a specific pathogen-free animal facility. The mice were randomly divided into three groups $(n=6)$, (I) Sham group, in which mice underwent laminectomy; (II) SCI + DMSO group, in which spinal cord contusion was conducted and equivalent DMSO solution to the $\mathrm{SCI}+\mathrm{ZO}$ group injected intraperitoneally per day. (III) $\mathrm{SCI}+\mathrm{ZO}$ group, in which contusion was conducted with injection of $2.5 \mathrm{mg} /$ $\mathrm{kg} \mathrm{ZO}$ solution. The first $\mathrm{ZO}$ administration was given at $1 \mathrm{~h}$ after SCI.

\section{Quantitative RT-PCR}

Total RNA was extracted from cells using TRIzol after LPS treatment for $12 \mathrm{~h}$. The concentration and purity of RNA were detected using UV-spectrophotometry (NanoDrop-2000, MA, USA) at $260 \mathrm{~nm}$ and $280 \mathrm{~nm}$. Reverse transcription of RNA using a Goldenstar ${ }^{\mathrm{TM}}$ RT6 cDNA Synthesis Kit (TsingKe, Beijing, China) in accordance with manufacturer's instruction, followed by the quantification using a SYBR Green Master (TsingKe). The target genes were normalized to GAPDH using the $\Delta \Delta^{\mathrm{Ct}}$ method. The primers were listed in Table 1.

\section{Western blot (WB)}

Protein extraction was carried out using a Total Protein Extraction Kit (KGP2100, KenGEN) according to the manufacturer's instructions after LPS treatment for $24 \mathrm{~h}$. After concentration determination by BCA method, equivalent protein was sequentially performed electrophoresis, transferring, and blocking. Protein was then probed with primary and secondary antibodies, and measured using an enhanced chemiluminescence system (Tanon, Shanghai, China). 
Table 1 Primer sequences of quantitative reverse transcription-polymerase chain reaction.

\begin{tabular}{|c|c|c|}
\hline \multirow{2}{*}{$\frac{\text { Oligo Name }}{\text { TNF-a (Ms) }}$} & \multicolumn{2}{|c|}{ Sequence (5' --------> $\left.3^{\prime}\right)$} \\
\hline & Forward & CTGAACTTCGGGGTGATCGG \\
\hline & Reverse & GGCTTGTCACTCGAATTTGAGA \\
\hline \multirow[t]{2}{*}{ IL-1 $(\mathrm{Ms})$} & Forward & GCAACTGTTCCTGAACTCAACT \\
\hline & Reverse & ATCTITTGGGTCCGTCAACT \\
\hline \multirow[t]{2}{*}{ MMP2 (Ms) } & Forward & CCTGGACCCTGAAACCGTG \\
\hline & Reverse & TCCCCATCATGGATTCGAGAA \\
\hline \multirow[t]{2}{*}{ MMP9 (Ms) } & Forward & GCAGAGGCATACTTGTACCG \\
\hline & Reverse & TGATGTTATGATGGTCCCACTTG \\
\hline \multirow[t]{2}{*}{ GAPDH (Ms) } & Forward & TGACCTCAACTACATGGTCTACA \\
\hline & Reverse & CTTCCCATTCTCGGCCTTG \\
\hline
\end{tabular}

\section{ELISA}

Tissues (4 mm length) were collected and homogenized on ice. Then the pro-inflammatory cytokines and chemokines from the supernatants were detected using the ELISA in accordance with the manufacturer's protocols.

\section{Trans-well assay}

Microglia $\left(2 \times 10^{5}\right.$ cells/ well $)$ were seeded into the upper chambers of trans-well inserts $(8 \mu \mathrm{m}$ membrane pore size; Corning, Corning, NY, USA) in FBS-free medium with the respective reagents, and the lower chambers were placed into a 24-well plate filled with $500 \mu \mathrm{L}$ complete medium containing LPS. After culturing for $24 \mathrm{~h}$, the unmigrated cells on the upper membrane were cleaned using a sterile swab. The migrated cells on the lower membrane were fixed using 4\% PFA for $30 \mathrm{~min}$ followed by staining with $0.1 \%$ crystal violet for $30 \mathrm{~min}$, the images were observed under a microscope.

\section{Histology and immunology staining}

Tissue collection and section manufacture were consistent with the previous description ${ }^{48}$. For histology, HE staining was performed on the sections according to the manufacturer's protocol. For IF, sections were incubated with the primary and secondary antibodies as described previously $^{48}$. Then sections were observed using a microscope system.

\section{Flow cytometry analysis (FCA)}

To measure the polarization of microglia, BV2 cells in each group were collected for $30 \mathrm{~min}$ incubation with F4_80-PE and iNOS-FITC at $4{ }^{\circ} \mathrm{C}$. Then cells were examined by flow cytometry (FACSVerse 8, BD Biosciences). Subsequently, the obtained data were analyzed using the FlowJo software (Version 7.6.1, Ashland, OR, USA).

\section{Behavioral assessment}

The locomotor of mouse hindlimb was examined for 4 weeks post SCI using the Basso

Mouse Scale (BMS). Each mouse was allowed to move in an open field while two experienced researchers observed their movements and scored at 3, 7, 14, 21, 28 days. The scores of two hind limbs were averaged.

\section{Statistical analysis}

Data from at least three independent biological experiments were exhibited as mean \pm SD. Analysis more than two groups was carried out using one-way or two-way ANOVA, and analysis for two groups was done through unpaired twotailed Student's $t$ tests. The graphs were drawn using the GraghPad Prism 6 software (San Diego, CA, USA). P-values $<0.05$ were considered statistically significant.

\section{Conclusion}

TAK1 inhibition attenuates neuroinflammation via blocking downstream MAPKs pathway in activated microglia. Moreover, downregulation of MAPKs pathway by blocking TAK1 activation reduced MMP2/9 expression and mitigated microglia migration, thus protecting neurological histology and function.

\section{Acknowledgements}

We thank the Medical School of Southeast University and Zhongda Hospital of Southeast University for providing us with helpful support.

\section{Author details}

${ }^{1}$ Spine Center, Zhongda Hospital of Southeast University, Nanjing, China. ${ }^{2}$ Department of Microbiology and Immunology, Medical School, Southeast University, Nanjing, China. ${ }^{3}$ Department of Orthopedics, First Affiliated Hospital of Nanjing Medical University, Nanjing, China. ${ }^{4}$ Department of Orthopedics, Nanjing First Hospital, Nanjing Medical University, Nanjing, China. ${ }^{5}$ Department of Orthopedics, Taizhou Clinical Medical School of Nanjing Medical University, Taizhou People's Hospital, Taizhou, China

\section{Author contributions}

S.J., Y.W., S.W., S.Y., J.C., R.K., M.X., J.B. and X.P. performed the experiments and analyzed data. Z.Q. wrote the manuscript and organized all the figures. X.H. and H.L. performed the investigation, methodology, project administration, writing review, and editing.

\section{Funding}

The present study has supported the Funding from the National Natural Science Foundation of China (81572190 to X.H.).

\section{Ethics approval}

Our SCl protocol was approved by the Animal Care and Utilization Institutional Committee of Southeast University.

Conflict of interest

The authors declare no competing interests.

\section{Publisher's note}

Springer Nature remains neutral with regard to jurisdictional claims in published maps and institutional affiliations. 
Received: 17 January 2021 Revised: 11 March 2021 Accepted: 13 April 2021 Published online: 08 May 2021

\section{References}

1. Mesquida-Veny F., Del R. J. \& Hervera A. Macrophagic and microglial complexity after neuronal injury. Prog. Neurobiol. 101970 (2020).

2. Greenhalgh, A. D., David, S. \& Bennett, F. C. Immune cell regulation of glia during CNS injury and disease. Nat. Rev. Neurosci. 21, 139-152 (2020).

3. David, S. \& Kroner, A. Repertoire of microglial and macrophage responses after spinal cord injury. Nat. Rev. Neurosci. 12, 388-399 (2011).

4. Zhou, X., He, X. \& Ren, Y. Function of microglia and macrophages in secondary damage after spinal cord injury. Neural Regen. Res. 9, 1787-1795 (2014).

5. Loane, D. J. \& Byrnes, K. R. Role of microglia in neurotrauma. Neurotherapeutics 7, 366-377 (2010)

6. Silva, N. A., Sousa, N., Reis, R. L. \& Salgado, A. J. From basics to clinical: a comprehensive review on spinal cord injury. Prog. Neurobiol. 114, 25-57 (2014).

7. Evaniew, N. et al. Methylprednisolone for the treatment of patients with acute spinal cord injuries: a propensity score-matched cohort study from a Canadian multi-center spinal cord injury registry. J. Neurotrauma 32, 1674-1683 (2015)

8. Ye, J. et al. Methylprednisolone inhibits the proliferation of endogenous neural stem cells in nonhuman primates with spinal cord injury. J. Neurosurg. Spine 29, 199-207 (2018).

9. Yamaguchi, $\mathrm{K}$. et al. Identification of a member of the MAPKKK family as a potential mediator of TGF-beta signal transduction. Science 270, 2008-2011 (1995).

10. Mihaly, S. R., Ninomiya-Tsuji, J. \& Morioka, S. TAK1 control of cell death. Cell Death Differ. 21, 1667-1676 (2014).

11. Sakurai, H. Targeting of TAK1 in inflammatory disorders and cancer. Trends Pharm. Sci. 33, 522-530 (2012).

12. Ajibade, A. A., Wang, H. Y. \& Wang, R. F. Cell type-specific function of TAK1 in innate immune signaling. Trends Immunol. 34, 307-316 (2013).

13. Guan, $H$. et al. Natural Xanthone alpha-Mangostin Inhibits LPS-Induced microglial inflammatory responses and memory impairment by blocking the TAK1/NF-KappaB signaling pathway. Mol. Nutr. Food Res. 64, e2000096 (2020).

14. Lei, C. Q. et al. USP19 Inhibits TNF-alpha- and IL-1 beta-triggered NF-KappaB activation by deubiquitinating TAK1. J. Immunol. 203, 259-268 (2019).

15. $\mathrm{Li}, \mathrm{X}$. et al. Tetrandrine regulates hepatic stellate cell activation via TAK1 and NF-kappaB signaling. Int Immunopharmacol. 36, 263-270 (2016).

16. Ge, B. et al. MAPKK-independent activation of p38alpha mediated by TAB1dependent autophosphorylation of p38alpha. Science 295, 1291-1294 (2002).

17. $\mathrm{Wu}, \mathrm{J}$. et al. Mechanism and in vitro pharmacology of TAK1 inhibition by (5Z)7-Oxozeaenol. ACS Chem. Biol. 8, 643-650 (2013).

18. Singh, A. K. et al. Suppression of monosodium urate crystal-induced inflammation by inhibiting TGF-beta-activated kinase 1-dependent signaling: role of the ubiquitin proteasome system. Cell Mol. Immunol. 18, 162-170 (2021).

19. Wang, R. et al. Transforming growth factor beta-activated kinase 1-dependent microglial and macrophage responses aggravate long-term outcomes after ischemic stroke. Stroke 51, 975-985 (2020).

20. Schwab, J. M., Zhang, Y., Kopp, M. A., Brommer, B. \& Popovich, P. G. The paradox of chronic neuroinflammation, systemic immune suppression, autoimmunity after traumatic chronic spinal cord injury. Exp. Neurol. 258, 121-129 (2014).

21. Popovich, P. G. \& Jones, T. B. Manipulating neuroinflammatory reactions in the injured spinal cord: back to basics. Trends Pharm. Sci. 24, 13-17 (2003).

22. Mortezaee, K., Khanlarkhani, N., Beyer, C. \& Zendedel, A. Inflammasome: its role in traumatic brain and spinal cord injury. J. Cell Physiol. 233, 5160-5169 (2018).

23. Sun, $X$. et al. Multiple organ dysfunction and systemic inflammation after spinal cord injury: a complex relationship. J. Neuroinflammation 13, 260 (2016).

24. Faden, A. I., Wu, J., Stoica, B. A. \& Loane, D. J. Progressive inflammationmediated neurodegeneration after traumatic brain or spinal cord injury. Br. J. Pharm. 173, 681-691 (2016).
25. Tan, S. et al. Hepatocyte-specific TAK1 deficiency drives RIPK1 kinasedependent inflammation to promote liver fibrosis and hepatocellular carcinoma. Proc. Natl Acad. Sci. USA 117, 14231-14242 (2020).

26. Santoro, R., Carbone, C., Piro, G., Chiao, P. J. \& Melisi, D. TAK-ing aim at chemoresistance: the emerging role of MAP3K7 as a target for cancer therapy. Drug Resist. Updat. 33-35, 36-42 (2017).

27. Li, J. et al. TAK1 inhibition attenuates both inflammation and fibrosis in experimental pneumoconiosis. Cell Discov. 3, 17023 (2017).

28. Kang, H. S. et al. Nuclear orphan receptor TAK1/TR4-deficient mice are protected against obesity-linked inflammation, hepatic steatosis, and insulin resistance. Diabetes 60, 177-188 (2011).

29. Kishida, S., Sanjo, H., Akira, S., Matsumoto, K. \& Ninomiya-Tsuji, J. TAK1-binding protein 2 facilitates ubiquitination of TRAF6 and assembly of TRAF6 with IKK in the IL-1 signaling pathway. Genes Cells 10, 447-454 (2005).

30. Kang, H. S. et al. TAB2 and TAB3 activate the NF-kappaB pathway through binding to polyubiquitin chains. Mol. Cell 15, 535-548 (2004).

31. Ninomiya-Tsuji, J. et al. The kinase TAK1 can activate the NIK-I kappaB as well as the MAP kinase cascade in the IL-1 signalling pathway. Nature $\mathbf{3 9 8}$ 252-256 (1999).

32. Zhu, H., Dai, R., Zhou, Y., Fu, H. \& Meng, Q. TLR2 ligand pam3CSK4 regulates MMP-2/9 expression by MAPK/NF-kappaB signaling pathways in primary brain microvascular endothelial cells. Neurochem. Res. 43, 1897-1904 (2018).

33. Zhang, $\mathrm{Y}$. et al. Tatarinan $\mathrm{N}$ inhibits osteoclast differentiation through attenuating NF-kappaB, MAPKs and $\mathrm{Ca}^{(2+)}$-dependent signaling. Int. Immunopharmacol. 65, 199-211 (2018).

34. Kyriakis, J. M. \& Avruch, J. Mammalian MAPK signal transduction pathways activated by stress and inflammation: a 10-year update. Physiol. Rev. 92, 689-737 (2012)

35. Arthur, J. S. \& Ley, S. C. Mitogen-activated protein kinases in innate immunity. Nat. Rev. Immunol. 13, 679-692 (2013).

36. Peluso, I., Yarla, N. S., Ambra, R., Pastore, G. \& Perry, G. MAPK signaling pathway in cancers: olive products as cancer preventive and therapeutic agents. Semin. Cancer Biol. 56, 185-195 (2019).

37. Chen, $\mathrm{H}$. et al. Silencing COX-2 blocks PDK1/TRAF4-induced AKT activation to inhibit fibrogenesis during skeletal muscle atrophy. Redox Biol. 38, 101774 (2020).

38. Qian, Z. et al. Excess administration of miR-340-5p ameliorates spinal cord injury-induced neuroinflammation and apoptosis by modulating the P38MAPK signaling pathway. Brain Behav. Immun. 87, 531-542 (2020).

39. Alaseem, A. et al. Matrix Metalloproteinases: a challenging paradigm of cancer management. Semin. Cancer Biol. 56, 100-115 (2019).

40. Chopra, S., Overall, C. M. \& Dufour, A. Matrix metalloproteinases in the CNS: interferons get nervous. Cell Mol. Life Sci. 76, 3083-3095 (2019).

41. Lee, E. J., Ko, H. M., Jeong, Y. H., Park, E. M. \& Kim, H. S. Beta-Lapachone suppresses neuroinflammation by modulating the expression of cytokines and matrix metalloproteinases in activated microglia. J. Neuroinflammation 12, 133 (2015).

42. Huang, Q. et al. IL-1beta-induced activation of $\mathrm{p} 38$ promotes metastasis in gastric adenocarcinoma via upregulation of AP-1/c-fos, MMP2 and MMP9. Mol. Cancer 13, 18 (2014).

43. Sun, Y. S. et al. Icariside II suppresses cervical cancer cell migration through JNK modulated matrix metalloproteinase-2/9 inhibition in vitro and in vivo. Biomed. Pharmacother. 125, 110013 (2020).

44. Qin, H. et al. PAD1 promotes epithelial-mesenchymal transition and metastasis in triple-negative breast cancer cells by regulating MEK1-ERK1/2MMP2 signaling. Cancer Lett. 409, 30-41 (2017).

45. Jiang, L. et al. Selective suppression of the JNK-MMP2/9 signal pathway by tetramethylpyrazine attenuates neuropathic pain in rats. J. Neuroinflammation 14, 174 (2017).

46. Lively, S. \& Schlichter, L. C. The microglial activation state regulates migration and roles of matrix-dissolving enzymes for invasion. J. Neuroinflammation 10, 75 (2013).

47. Kehl, S. R. et al. TAK1 converts sequestosome 1/p62 from an autophagy receptor to a signaling platform. EMBO Rep. 20, e46238 (2019).

48. Hong, X. et al. Treatment with 5-methoxytryptophan attenuates microgliainduced neuroinflammation in spinal cord trauma. Int. Immunopharmacol. 88 106988 (2020). 